\title{
LIE ALGEBRAS ASSOCIATED WITH GENERALIZED CARTAN MATRICES
}

\author{
BY R. V. MOODY
}

Communicated by Charles W. Curtis, October 3, 1966

1. Introduction. If $\mathfrak{R}$ is a finite-dimensional split semisimple Lie algebra of rank $n$ over a field $\Phi$ of characteristic zero, then there is associated with $\mathfrak{R}$ a unique $n \times n$ integral matrix $\left(A_{i j}\right)$-its Cartan matrix-which has the properties

M1.

$$
\begin{aligned}
& A_{i i}=2, \quad i=1, \cdots, n, \\
& \text { M2. } \quad A_{i j} \leqq 0, \quad \text { if } i \neq j \text {, }
\end{aligned}
$$$$
\text { M3. } \quad A_{i j}=0, \quad \text { implies } A_{j i}=0 \text {. }
$$

These properties do not, however, characterize Cartan matrices.

If $\left(A_{i j}\right)$ is a Cartan matrix, it is known (see, for example, [4, pp. VI-19-26]) that the corresponding Lie algebra, $\mathfrak{R}$, may be reconstructed as follows: Let $e_{i}, f_{i}, h_{i}, i=1, \ldots, n$, be any $3 n$ symbols. Then $\mathfrak{R}$ is isomorphic to the Lie algebra $\tilde{\mathfrak{l}}\left(\left(A_{i j}\right)\right)$ over $\delta$ defined by the relations

$$
\begin{aligned}
& {\left[h_{i} h_{j}\right]=0,} \\
& \left.\begin{array}{l}
{\left[e_{i} f_{j}\right]=\delta_{i j} h_{i},} \\
{\left[e_{i} h_{j}\right]=A_{j i} e_{i}, \quad\left[f_{i} h_{j}\right]=-A_{j i} f_{i},}
\end{array}\right\} \text { for all } i \text { and } j \\
& \left.\begin{array}{l}
e_{i}\left(\operatorname{ad} e_{j}\right)^{-\Lambda_{j i}+1}=0, \\
f_{i}\left(\operatorname{ad} f_{j}\right)^{-A_{j i}+1}=0,
\end{array}\right\} \text { if } i \neq j .
\end{aligned}
$$

In this note, we describe some results about the Lie algebras $\tilde{\mathbb{P}}\left(\left(A_{i j}\right)\right)$ when $\left(A_{i j}\right)$ is an integral square matrix satisfying M1, M2, and M3 but is not necessarily a Cartan matrix. In particular, when the further condition of $\$ 3$ is imposed on the matrix, we obtain a reasonable (but by no means complete) structure theory for $\tilde{\mathfrak{L}}\left(\left(A_{i j}\right)\right)$.

2. Preliminaries. In this note, $\Phi$ will always denote a field of characteristic zero. An in tegral square matrix satisfying M1, M2, and M3 will be called a generalized Cartan matrix, or g.c.m. for short. $Z$ will denote the integers, and in any Lie algebra we will use the symbol $\left[l_{1}, l_{2}, \cdots, l_{n}\right]$ to denote the product $\left[\cdots\left[\left[l_{1} l_{2}\right] \cdots\right] l_{n}\right]$.

1 These results were obtained in my dissertation at the University of Toronto under the supervision of Professor M. J. Wonenburger. 
Let $\left(A_{i j}\right)$ be a g.c.m. and let $\mathfrak{R} \tilde{\mathbb{R}}\left(\left(A_{i j}\right)\right)$ be the Lie algebra over $\Phi$ which is obtained by using the method of $\S 1$. Many of the customary features of finite-dimensional split semisimple Lie algebras appear in $\mathfrak{l}$. For example, $\mathbb{R}$ is graded by $Z \times \cdots \times Z$ (taken $n$ times), and if we denote the subspace of elements of degree $\left(d_{1}, \cdots, d_{n}\right)$ by $\mathfrak{R}\left(d_{1}, \cdots, d_{n}\right)$, we find that $\tilde{\mathbb{S}} \equiv \mathbb{R}(0, \cdots, 0)$ is the subspace generated by $h_{1}, \cdots, h_{n}$, and $\&\left(d_{1}, \cdots, d_{n}\right)=(0)$ unless $d_{1}, \cdots, d_{n}$ are all nonnegative or all nonpositive. Furthermore, if $d_{1}, \cdots, d_{n}$ are all nonnegative (respectively all nonpositive) and not all zero, then $\mathfrak{R}\left(d_{1}, \cdots, d_{n}\right)$ is spanned by the elements of the type $\left[e_{i_{1}}, \cdots, e_{i_{r}}\right]$ (respectively $\left[f_{i_{1}}, \cdots, f_{i_{r}}\right]$ ) where each $e_{j}$ (respectively $f_{j}$ ) appears precisely $\left|d_{j}\right|$ times.

Let $\alpha$ be an $n$-dimensional vector space over $\Phi$ with a basis $\alpha_{1}$, $\cdots, \alpha_{n}$, and define a mapping, $\sim$, of $\propto$ into $\tilde{\mathbb{F}}^{*}$, the dual space of $\tilde{\mathfrak{E}}$, by putting $\tilde{\alpha}_{i}\left(h_{j}\right)=A_{j i}$ for $i, j=1, \cdots, n$. Then, if $a \in \mathfrak{R}\left(d_{1}, \cdots, d_{n}\right)$, $\left[\begin{array}{ll}a & h\end{array}\right]=\left(\sum_{i=1}^{n} d_{i} \alpha_{i}\right) \sim(h) a$ for all $h \in \widetilde{\mathfrak{W}}$. If $\mathfrak{R}\left(d_{1}, \cdots, d_{n}\right) \neq(0)$, we call $\beta=\sum d_{i} \alpha_{i}$ a root and write $\Re_{\beta}$ for $\mathcal{R}\left(d_{1}, \cdots, d_{n}\right)$. We use the words positive and negative for the nonzero roots in the usual way.

A g.c.m., $\left(A_{i j}\right)$, is said to be decomposable if, after a suitable permutation of the rows together with the corresponding permutation of the columns, it takes a diagonal block form. Clearly, any block obtained in this manner is a g.c.m. $\left(A_{i j}\right)$ is called indecomposable if it is not decomposable. If $\left(A_{i j}\right)$ decomposes into indecomposable blocks $B_{1}, \cdots, B_{k}$, then $\tilde{\mathbb{R}}\left(\left(A_{i j}\right)\right) \cong \tilde{\mathbb{R}}\left(B_{1}\right) \times \cdots \times \tilde{\mathbb{R}}\left(B_{k}\right)$. Consequently, we restrict our attention to indecomposable g.c.m.'s.

At this point, we impose a strong condition on our g.c.m.'s. Further results on the algebras $\widetilde{\mathfrak{Q}}\left(\left(A_{i j}\right)\right)$ when no further restrictions are placed on the matrix are discussed in a forthcoming note in this journal by Daya-Nand Verma.

3. G.C.M.'s of Type (1) and (2), and their classification. Let $\left(A_{i j}\right)$ be an indecomposable g.c.m. which satisfies the following condition: If $\xi_{1}, \cdots, \xi_{n}$ are any nonnegative rational numbers such that $\sum_{i=1}^{n} \xi_{i} A_{j i} \leqq 0, j=1, \cdots, n$, then $\sum_{i=1}^{n} \xi_{i} A_{j i}=0, j=1, \cdots, n$. Such a matrix will be called a g.c.m. of type (2) if there exist nonnegative rationals $\xi_{1}, \cdots, \xi_{n}$, not all zero, such that $\sum_{i=1}^{n} \xi_{i} A_{j i}=0, j=1$, ...,n, and a g.c.m. of type (1) otherwise. From now on all g.c.m.'s will be of type (1) or (2).

A real, symmetric $n \times n$ matrix is called an $a$-form, $[1$, p. 175], if every entry off the diagonal is nonpositive. It is called connected if it is indecomposable in the sense above. 
THEOREM 1. If $\left(A_{i j}\right)$ is a g.c.m. of type (1) or (2), then there exist unique positive rational numbers $\omega_{1}, \cdots, \omega_{n}$ such that (i) $\omega_{i} A_{i j}=\omega_{j} A_{j i}$ for $i, j=1, \cdots, n$, and (ii) $\left\{\omega_{i} A_{i i} \mid i=1, \cdots, n\right\}$ is a set of integers with no common factor. The matrix $\left(a_{i j}\right) \equiv\left(\omega_{i} A_{i j}\right)$ is a connected a-form. It is positive definite or positive semidefinite according as $\left(A_{i j}\right)$ is of type (1) or (2), and $A_{i j}=2 a_{i j} / a_{i i}$. Conversely, if $\left(a_{i j}\right)$ is a positive definite (respectively positive semidefinite) connected a-form such that $A_{i j}$ $\equiv 2 a_{i j} / a_{i i}$ is an integer for each $i$ and $j$, then $\left(A_{i j}\right)$ is a g.c.m. of type (1) (respectively type (2)).

Let $\alpha_{0}$ be the rational space spanned by $\alpha_{1}, \cdots, \alpha_{n}$, and define a bilinear form $\sigma$ on $a_{0}$ by $\sigma\left(\alpha_{i}, \alpha_{j}\right)=a_{i j} . \sigma$ is positive definite or positive semidefinite according as $\left(A_{i j}\right)$ is of type (1) or (2), and in the latter case, $Q_{0}$ contains precisely one isotropic line, and it is the radical of $\sigma$.

On $a_{0}$ we define $S_{i}$ to be the reflection determined by the hyperplane orthogonal to $\alpha_{i}(i=1, \cdots, n)$. The group $W$ generated by the $S_{i}$ is called the Weyl group of $\tilde{\mathfrak{l}}\left(\left(A_{i j}\right)\right)$. Let $\Gamma=\left\{\beta \in a_{0} \mid \beta=\alpha_{j} T\right.$ for some $j=1, \cdots, n$, and some $T \in W\}$.

Theorem 2. (i) $A_{i j} A_{j i}=0,1,2,3$, or 4 for all $i, j$.

(ii) $\left(S_{i} S_{j}\right)^{p_{i j}}=1$ where

$$
\begin{aligned}
p_{i j} & =\pi /\left(\operatorname{Cos}^{-1}\left(\left(A_{i j} A_{j i}\right)^{1 / 2} / 2\right)\right), & & \text { if } i \neq j \\
& =1, & & \text { if } i=j .
\end{aligned}
$$

(iii) $a_{i j} /\left(a_{i i} a_{j j}\right)^{1 / 2}=-\cos \left(\pi / p_{i j}\right)$ for all $i$ and $j$.

The positive definite and positive semidefinite matrices of the type $\left(-\cos \left(\pi / p_{i j}\right)\right)$, where the $p_{i j}$ are integers such that $p_{i i}=1, p_{i j}>1$ if $i \neq j$, and $p_{i j}=p_{j i}$ for all $i$ and $j$, have already been classified [1, Chapter 11], and Theorem 2 (iii) provides us with a link by which we may classify the g.c.m.'s of type (1) and (2). These matrices may be diagrammatically described in the way customary for Cartan matrices - namely by drawing a dot for each number $1, \cdots, n$, joining the $i$ th and $j$ th dots by $A_{i j} A_{j i}$ lines, and writing the weight $\sigma\left(\alpha_{i}, \alpha_{i}\right)=a_{i i}$ over the $i$ th dot. The g.c.m.'s of type (1) turn out to be precisely the indecomposable Cartan matrices, and the Lie algebras $\tilde{\mathfrak{R}}\left(\left(A_{i j}\right)\right)$ obtained from them are, of course, the corresponding finite-dimensional split simple Lie algebras over $\Phi$. The diagrams for the type (2) matrices are, except for the weights, basically those given in $[2$, p. 142]. The only change required is the replacement of any line with a number $m$ appearing over it by $4 \cos ^{2}(\pi / m)$ lines. Each type (2) matrix is designated by the letter attached to its diagram by 
Coxeter. As in the case of Cartan matrices, different matrices may have the same diagram with only the weight distribution differing. We use a second subscript to distinguish matrices with the same diagram. With this notation, the complete list of type (2) g.c.m.'s is: $P_{n}, n>2 ; S_{n, 1}, S_{n, 2}, n>2 ; R_{n, 1}, R_{n, 2}, R_{n, 8}, n>2 ; Q_{n}, n>4 ; T_{7}, T_{8}, T_{9}$; $U_{5,1}, U_{5,2} ; V_{\mathbf{3}, 1}, V_{3,2} ; W_{2,1}, W_{2,2.2}$

REMARK. If $\left(A_{i j}\right)$ is a g.c.m. of type (1), then $W$ is the group defined by the relations of Theorem 2 (ii). We do not know whether this result holds for the g.c.m.'s of type (2).

4. The structure theory. If $\left(A_{i j}\right)$ is of type (2), then $\tilde{\mathbb{R}}\left(\left(A_{i j}\right)\right)$ has a centre, $\mathfrak{C}$. $\mathfrak{C}$ is a homogeneous ideal, and $\mathfrak{R}\left(\left(A_{i j}\right)\right) \equiv \tilde{\mathbb{R}}\left(\left(A_{i j}\right)\right) / \mathfrak{C}$ still decomposes in to a direct sum of root spaces. The image, $\mathfrak{S}$, of $\tilde{\mathfrak{E}}$ in $\mathfrak{R}\left(\left(A_{i j}\right)\right)$ is of dimension $n-1$. $\mathfrak{R}\left(\left(A_{i j}\right)\right)$ has no centre. We of ten designate $\mathfrak{R}\left(\left(A_{i j}\right)\right)$ by the symbol for the matrix $\left(A_{i j}\right)$. The algebras $\mathfrak{R}\left(\left(A_{i j}\right)\right)$, when $\left(A_{i j}\right)$ is a g.c.m. of type (2), are called tiered algebras

THEOREM 3. If $\&$ is tiered, then $\beta \in Q_{0}$ is a nonisotropic root if and only if $\beta \in \Gamma$, and for such roots $\operatorname{dim} \Omega_{\beta}=1$. There exists a positive isotropic root $\zeta$ such that the set of isotropic roots is precisely $Z \zeta$. There exists a positive integer $r$ such that if $\beta$ is a root, then $\{\beta+Z r \zeta\}$ are all roots (clearly, then, $\mathbb{R}$ is infinite dimensional). The minimum $r$ for which this is true is called the tier number of $\mathbb{R}$, and $\mathbb{R}$ is said to be r-tiered. The tier number is always 1, 2, or 3. In fact, the algebras $P_{n}, S_{n, 1}$, $R_{n, 1}, Q_{n}, T_{7}, T_{8}, T_{9}, U_{5,1}, V_{3,1}$, and $W_{2,1}$ are 1-tiered, and the remaining ones are 2-tiered with the exception of $V_{\mathbf{3}, 2}$ which is 3-tiered.

THEOREM 4. If $\&$ is $r$-tiered, and is treated as an $\&$-module relative to its adjoint representation, then there exists an \&-module automorphism of $\mathfrak{\Omega}$ (denoted by') such that $\mathfrak{R}_{\beta} \rightarrow \mathfrak{\Omega}_{\beta+r\}}$ for all roots $\beta$.

The mapping of Theorem 4 is called the shift mapping, and plays a fundamental role in proving the remaining theorems. If $l \in R$, we define $l^{(i)}, i=0,1,2, \ldots$ inductively by $l^{(0)}=l, l^{(i)}=\left(l^{(i-1)}\right)^{\prime}$ for $i>0$.

THEOREM 5. If $\Im$ is a nonzero ideal of the tiered Lie algebra $\mathfrak{R}$, then $\Im$ is generated by a single element of the form $\sum_{i=0}^{s} \lambda_{i} h_{1}^{(i)}$ with $\lambda_{0} \neq 0$, and $\lambda_{s}=1$. The correspondence between nonzero ideals and elements of this type is bijective.

Let $\Phi\langle x\rangle$ denote the ring of polynomials of the form $\sum_{i=-\infty}^{\infty} \mu_{i} x^{i}$ with almost all of the $\mu_{i}=0$.

${ }^{2}$ I am grateful to Professor G. B. Seligman for pointing out that the matrices of type $W$ were omitted in my original classification. 
THEOREM 6. The lattice of ideals of a tiered Lie algebra over $\Phi$ is isomorphic to the lattice of ideals of $\Phi\langle x\rangle$.

If $\mu \in \Phi, \mu \neq 0$, the ideal generated by $h_{1}-\mu h_{1}^{(1)}$ is maximal. We denote its quotient in $\&$ by $\mathcal{R}(\mu)$.

THEOREM 7. $\mathfrak{R}(\mu)$ is finite-dimensional and central simple. $\operatorname{dim} \mathfrak{R}(\mu)$ is the same for all nonzero $\mu$.

The next theorem tells us that the 1-tiered algebras are not really anything new.

THEOREM 8. If $\mathbb{R}$ is 1 -tiered, then $\mathbb{R} \cong \mathbb{R}(1) \otimes_{\Phi} \Phi\langle x\rangle$. The relation between $\mathbb{R}(1)$ and $\left(A_{i j}\right)$ is given by the table:

\begin{tabular}{|ll||lc|}
\hline$\left(A_{i j}\right)$ & $\mathfrak{L}(1)$ & $\left(A_{i j}\right)$ & $\mathfrak{Q}(1)$ \\
\hline$P_{n}$ & $A_{n-1}$ & $T_{8}$ & $E_{7}$ \\
$S_{n, 1}$ & $B_{n-1}$ & $T_{9}$ & $E_{8}$ \\
$R_{n, 1}$ & $C_{n-1}$ & $U_{8,1}$ & $F_{4}$ \\
$Q_{n}$ & $D_{n-1}$ & $V_{8,1}$ & $G_{2}$ \\
$T_{7}$ & $E_{6}$ & $W_{2,1}$ & $A_{1}$ \\
\hline
\end{tabular}

THEOREM 9. If $\left(A_{i j}\right)$ is an $n \times n$ g.c.m. of type (2), and $\operatorname{dim} \mathfrak{R}(\mu)=m$ ' and if $\left(\mu_{1} \mu_{2}^{-1}\right)^{m-n+1}$ is a nonsquare in $\Phi$, then $\mathbb{R}\left(\mu_{1}\right) \neq \mathbb{R}\left(\mu_{2}\right)$.

This theorem is difficult to apply because we do not know the dimension of $\mathfrak{R}(\mu)$ in general. However, a low dimensional survey reveals that if $\Phi$ contains nonsquares there are at least two nonisomorphic algebras of each of the forms $S_{5,2}(\mu), R_{4,2}(\mu), R_{5,2}(\mu)$, and $R_{4,8}(\mu)$, and they are of the types (in the sense of $\left[3\right.$, p. 299]) $A_{7}, D_{4}$, $D_{5}$, and $A_{6}$ respectively.

Added in proof: The problem raised in the "Remark" has been answered in the affirmative.

\section{BIBLIOGRAPHY}

1. H. S. M. Coxeter, Regular polytopes, 2nd ed., Macmillan, New York, 1963.

2. - Generators and relations for discrete groups, 2nd ed., Ergebnisse der Mathematik und ihrer Grenzgebiete, Band 14, Springer-Verlag, New York, 1965.

3. N. Jacobson, Lie algebras, Interscience Tracts 10, Interscience, New York, 1962.

4. J.-P. Serre, Algèbres de Lie semi-simples complexes, Benjamin, New York, 1966.

University of Saskatchewan 